\title{
Ancient animal microRNAs and the evolution of tissue identity
}

\author{
Foteini Christodoulou ${ }^{1}$, Florian Raible ${ }^{1,2, \dagger}$, Raju Tomer $^{1}$, Oleg Simakov ${ }^{1}$, Kalliopi \\ Trachana $^{2}$, Sebastian Klaus ${ }^{1}, \dagger$, Heidi Snyman ${ }^{1}$, Gregory J. Hannon ${ }^{3}$, Peer Bork ${ }^{2}$, and Detlev \\ Arendt ${ }^{1}$ \\ ${ }^{1}$ Developmental Biology Unit, European Molecular Biology Laboratory, D-69117 Heidelberg, \\ Germany. \\ ${ }^{2}$ Computational Biology Unit, European Molecular Biology Laboratory, D-69117 Heidelberg, \\ Germany. \\ ${ }^{3}$ Cold Spring Harbor Laboratory, 1 Bungtown Road, Cold Spring Harbor, New York 11724, USA.
}

\section{Abstract}

The spectacular escalation in complexity in early bilaterian evolution correlates with a strong increase in the number of microRNAs 1,2 . To explore the link between the birth of ancient microRNAs and body plan evolution, we set out to determine the ancient sites of activity of conserved bilaterian microRNA families in a comparative approach. We reason that any specific localization shared between protostomes and deuterostomes (the two major superphyla of bilaterian animals) should probably reflect an ancient specificity of that microRNA in their last common ancestor. Here, we investigate the expression of conserved bilaterian microRNAs in Platynereis dumerilii, a protostome retaining ancestral bilaterian features ${ }^{3,4}$, in Capitella, another marine annelid, in the sea urchin Strongylocentrotus, a deuterostome, and in sea anemone Nematostella, representing an outgroup to the bilaterians. Our comparative data indicate that the oldest known animal microRNA, miR-100, and the related miR-125 and let-7 were initially active in neurosecretory cells located around the mouth. Other sets of ancient microRNAs were first present in locomotor ciliated cells, specific brain centres, or, more broadly, one of four major organ systems: central nervous system, sensory tissue, musculature and gut. These findings reveal that microRNA evolution and the establishment of tissue identities were closely coupled in bilaterian evolution. Also, they outline a minimum set of cell types and tissues that existed in the protostome-deuterostome ancestor.

Deep sequencing of Platynereis small RNAs identified 34 microRNA families common to protostomes and deuterostomes (Supplementary Fig. 1, Supplementary Table 1), in accordance with recent studies ${ }^{2}$. To investigate the temporal and spatial localization profile of these

(O2010 Macmillan Publishers Limited. All rights reserved

Correspondence and requests for materials should be addressed to D.A. (arendt@embl.de).

†Present addresses: Max F. Perutz Laboratories, Campus Vienna Biocenter, Dr. Bohr-Gasse 9/4, A-1030 Vienna, Austria (F.R.); J. W. Goethe - Universität Frankfurt Biologie-Campus, Siesmayerstrasse 70-72, D-60323 Frankfurt, Germany (S.K.).

Supplementary Information is linked to the online version of the paper at www.nature.com/nature.

Author Contributions F.C. initiated the project, cloned Platynereis small RNAs, characterized the temporal and spatial expression of ancient miRNAs and their targets, coordinated the collaborations and wrote the paper. F.R. analysed and evaluated the Solexa sequencing data. R.T. assembled the $3^{\prime}$ UTRs of targets from Platynereis ESTs and provided riboprobes. O.S. did the SNP and miRNA::target coexpression analysis. K.T. performed target predictions under the supervision of P.B., and S.K characterized foxa2 expression. H.S. generated probes for targets in situ screen. G.J.H. hosted the small RNA cloning and sequencing. D.A. analysed comparative miRNA expression, provided ideas and strategies and wrote the paper. All authors discussed the results and commented on the manuscript.

Author Information Sequences for Platynereis miRNA primary transcripts pri-miR-100/let-7, pri-miR-12/216 and pri-miR-183/263 were deposited in the GenBank database with accession numbers FJ838789.1, FJ838790.1 and GU224283, respectively. Reprints and permissions information is available at www.nature.com/reprints. The authors declare no competing financial interests. 
conserved bilaterian microRNAs, whole mount in situ hybridization (WMISH) was conducted using locked nucleic acids as probes. In all cases, localization of mature Platynereis microRNAs was spatially restricted and almost exclusively occurred in actively differentiating tissues (Supplementary Fig. 2).We also analysed tissue-specific expression of predicted targets (Supplementary Fig. 3 a, b and Supplementary Table 2) and identified a subset of tissues in which microRNAs were less frequently co-expressed with their predicted targets than expected to occur by chance, whereas other tissues showed the opposite trend (Supplementary Table 3 $\mathrm{a}, \mathrm{b}$ and Supplementary Data), indicating different modes of involvement of the conserved bilaterian microRNAs in regulating the establishment and/or maintenance of tissue identity ${ }^{5}$.

We found the oldest conserved microRNA, miR-100, shared by cnidarians and bilaterians ${ }^{1,2}$, highly specifically localized in two small groups of cells of the larval foregut (Fig. 1a, e). These cells also expressed let-7 (Fig. 1b, f) and miR-125 (Fig. 1c), which play a conserved role in developmental timing ${ }^{6-8}$, and miR-375 (Fig. 1d), demarcating foregutrelated, neurosecretory/ endocrine cell populations of vertebrate pituitary and pancreas ${ }^{9}$. Marker gene analysis revealed that these cells are differentiated secretory neurons (Supplementary Fig. 4c). As in fly ${ }^{7,8}$, PlatynereismiR-100 and let-7 are processed froma single polycistronic transcript (Supplementary Table 4) that in many bilaterians also includes miR-125 (refs 7,8). Since miR-100, miR-125 and let-7 are expressed much more broadly in fly 8 and vertebrate 10 (Supplementary Table 5), we challenged evolutionary conservation of the highly specific Platynereis pattern by investigating the expression of miR-100 in a cnidarian, the sea anemone Nematostella. miR-100 was exclusively detected in single cells located around the pharynx anlage (Fig. $1 \mathrm{~g}$ and Supplementary Fig. 5a, b), demarcated by brachyury ${ }^{11}$ and foxa expression (blue, red and yellow in Fig. 1h). Intriguingly, miR-100, brachyury 12 and foxa 2 also colocalize in the Platynereis foregut (Fig. 1i; blue, red and yellow in Fig. 1j). These findings indicate that early in animal evolution miR-100 was active in a small population of cells located around a digestive opening. This expression was inherited by let- 7 and by miR- 125 once they evolved in the bilaterian stem line, to expand into other tissues only later in evolution. Corroborating this, expression of miR-100, miR-125 and let-7 was similarly restricted to foregut tissue in Capitella (Fig. 1k, data not shown) and in the sea urchin Strongylocentrotus (Fig. 11 and Supplementary Fig. 5c, e).

Locomotor ciliary bands are characteristic for the swimming larvae of marine protostomes and deuterostomes ${ }^{13}$. In Platynereis, miR-29, miR-34 and miR-92 demarcated the ciliary bands from early larval stages onwards (Fig. 2a-f) and were also detected in motile ciliated cells of the apical organ and later in the medial head region (Fig. 2a-e). While expression was similar for all 'ciliary' microRNAs at early larval stages, partly complementary patterns were observed in the developing young worm (arrowheads in Fig. 2d, e). Capitella showed a similar localization of these microRNAs to ciliary bands (Fig. 2h and Supplementary Fig. 6a, b). We also investigated the sea urchin pluteus larva and indeed found miR-92 expressed in the ciliary bands (Fig. 2i) and miR-92 and miR-34 in motile ciliated cells lining the foregut and stomach (Supplementary Fig. 5g, h). The similar specific affiliation of these microRNAs to motile ciliated cells in both protostome and deuterostome larvae is best explained by evolutionary conservation.

Two sets of conserved bilaterian microRNAs showed localized expression in distinct parts of the brain. For miR-7 (ref. 4), miR-137 and miR-153 we observed very similar expression in the dorso-medial neurosecretory tissue of the Platynereis brain (Fig. 3a-d) comprising differentiated vasotocinergic and FMRFamidergic ${ }^{4}$ as well as serotonergic neurons (Fig. 3e). The same microRNAs showed brain-restricted expression in Capitella (Supplementary Fig. $6 \mathrm{c}-\mathrm{e})$ and have also been reported to show spatially localized expression in the zebrafish brain, including neurosecretory brain parts of the hypothalamus ${ }^{14}$ (Supplementary Table 5). Our comparative data thus indicate that these three microRNAs co-evolved in neurosecretory brain 
tissue. A conserved pair of complementary microRNAs, miR-9 and miR-9*/miR-131 (ref.

15) also showed highly restricted expression in the annelid brain, in two ventro-lateral sets of differentiated neurons (Fig. 3f-h and Supplementary Fig. 6f). In mouse, miR-9 and miR-9*/ miR-131 are detected broadly in neuronal precursors but among all differentiated neurons are expressed only in the telencephalon ${ }^{15}$, comprising olfactory brain centres. In Platynereis, the most apical cells expressing mature miR-9 and miR- $9 *$ were located at the base of the antennae, a pair of head appendages considered to be chemosensory sense organs (Fig. 3h). This indicates that miR-9 and miR-9* may ancestrally locate to neurons involved in olfactory/chemosensory information processing. As in vertebrates, both miR-9 and miR-9* are detected in relatively high abundance in Platynereis and thus represent a pair of complementary microRNAs conserved in bilaterians ${ }^{2}$.

Other conserved bilaterian microRNAs were expressed more broadly in one of four major organ systems, representing the central nervous system, peripheral sensory tissue, musculature or gut. For each of these groups, expression of individual microRNAs was largely overlapping, but at the same time complementary to those of other groups. miR-124 (Fig. 4a, d), known to maintain neuronal identity in the vertebrates 14,16 and expressed in developing central nervous system (CNS) in fly ${ }^{17}$ and planarian 18 , was found in differentiating neurons in the brain and ventral nerve cord in Platynereis. miR-71, lost in vertebrates and insects ${ }^{2}$ and restricted to CNS and parenchyma in planarians ${ }^{18}$, is also nervous system-specific in Platynereis (Fig. 4b, c and Supplementary Fig. 6h). miR-8 proved to be an excellent marker for differentiating sensory organs, including eyes, antennae, palpae and sensory organs of the parapodia ${ }^{19}$, covering both neural and non-neural tissue (Fig. 4e and Supplementary Fig. 6j). A sensory tissue affiliation is likely to be evolutionarily ancient for this microRNA since the related miR-200a, miR-200b and miR-141 likewise show restricted expression in sensory organs such as nose and lateral line in the vertebrates ${ }^{10}$ (Supplementary Table 5). miR-183 and miR-263, clustered in the Lottia genome and processed from the same transcript in Platynereis (Supplementary Table 4), also showed a conserved affiliation with sensory organ differentiation (Fig. 4f, g and Supplementary Fig. 6i), as previously reported for other bilaterians ${ }^{14,17}$. Notably, the expression of the sensory tissue-specific miR-183 and of the CNS-specific miR-124 was mutually exclusive (compare Fig. 4d, h). In fly and vertebrate, miR-1 is active during muscular differentiation ${ }^{10,17}$. In Platynereis, miR-1 and miR-133, clustered in vertebrates and in molluscs (Supplementary Table 4), showed almost identical expression in the differentiating musculature (Fig. 4i, k, l). In vertebrates, these microRNAs are robustly induced upon myotube differentiation concomitant with reduced expression of their target messengers $16,20,21$. Similar musculature-specific expression was observed for miR-22 (Fig. 4j) that together with miR-1 and miR-133 has been reported to have myoD and myogenin upstream binding sites in the vertebrates $^{20}$. Finally, miR-12, miR-216 and miR-283 showed identical expression in the differentiating midgut at 5 days post fertilization (d.p.f.) (Fig. 4m-p). miR-12 clusters with miR-216 in Platynereis and in Lottia ${ }^{22}$ and with miR-283 in Drosophila ${ }^{17}$ (Supplementary Table 4), indicating that these three microRNAs evolved from the same precursor gene. In the vertebrates, expression of miR-216 is characteristic of pancreatic tissue ${ }^{23}$ and targets of $m i R-216$ are expressed at lower levels in pancreatic than in other tissue ${ }^{21}$, indicating that the ancient site of activity of the miR-12/-216/-283 precursor had indeed been the gut.

Our results indicate that the conserved bilaterian microRNAs evolved in a strictly tissuespecific context. Corroborating this, brain-or musculature-specific microRNAs that we found in Platynereis show similar specificity in fish, human and mouse expression profiling, with high confidence (Supplementary Table 5). Although we cannot exclude that other expression sites initially existed for some of these microRNAs, these would have been lost in subsequent evolution in multiple lineages. At the present state of analysis, we can only speculate about the tissue-specific ancient roles of these microRNAs, by extrapolating from observations in other animal models. For example, miR-100 and the related miR-125 and let-7 may have acquired 
an ancient role in developmental timing: The very late onset of let-7 expression at 5 d.p.f. in Platynereis (before settlement) is consistent with earlier observations in nematode ${ }^{6}$, fly $^{7}, 8$, mollusc24, zebrafish ${ }^{24}$ and in another annelid ${ }^{24}$, indicative of a role in the control of late developmental transitions ${ }^{6-8,24}$. With time, such role may then have spread to other tissues, such as target tissue differentiating in the course of metamorphosis, as observed in fly 7,8 and nematode ${ }^{6,24}$. Similarly, the affinity of miR-29, miR-34 and miR-92 to motile ciliated cells sheds new light on the evolution of ventricular neuron types expressing these microRNAs in the vertebrate $\mathrm{CNS}^{14}$ (Supplementary Table 5), some of which are known to bear motile cilia $^{25}$. A comparison of targets in slow-evolving species should reveal the functional evolution of the conserved bilaterian microRNAs and also allow understanding why in many cases these microRNAs were co-opted by new tissues that did not express them before.

The identification of ancient expression sites for conserved bilaterian microRNAs implies that these microRNA-defined tissues existed in the protostome-deuterostome ancestor (Supplementary Fig. 1). Our data indicate that it possessed a miR-124+ central nervous tissue as opposed to miR-8/-183/-263+ peripheral sensory nervous tissue ${ }^{19}$, consistent with the notion that nervous system centralization predated the protostome/deuterostome ancestor ${ }^{19}$. The ancient brain comprised miR-7+, miR-137+ and miR-153+ neurosecretory parts, as recently suggested 4 and miR-9/9*+ parts that may relate to sensory information processing. Our data also indicate the existence of miR-1/-22/-133+ body musculature, in line with the proposed conservation of somatic muscle cell types ${ }^{19}$, and of a miR-12/-216/-283+ gut. Finally, the protostome/deuterostome ancestors possessed miR-100/-125/let-7/+ neurosecretory cells along the mouth and miR-29, miR-34, miR-92+ motile ciliated cells, possibly forming part of ot $x+$ larval ciliary bands 12,26 . We have thus established microRNAs as an important new tool for reconstructing ancient animal body plans at important evolutionary nodes, focusing here on the protostome-deuterostome divergence. More complete inventories of microRNAsyielding a refined picture of gains (and losses) of microRNAs in the diverging lineages — and concomitant expression analysis will allow expanding this approach to other key events of animal evolution.

\section{METHODS SUMMARY}

\section{Platynereis and Capitellawhole mount in situ hybridization with locked nucleic acids probes}

Whole mount in situ hybridizations (WMISH) were performed by modifying fish and Platynereis established protocols ${ }^{10,27}$. Use of a more stringent hybridization mix with $70 \%$ formamide allowed lowering the hybridization temperature down to $37{ }^{\circ} \mathrm{C}$ to successfully visualize the expression of any microRNA tested (regardless of the locked nucleic acids' $T_{\mathrm{m}}$ ). The same in situ protocol was used for the hybridization of long DIG-11-UTP-labelled RNA probes.

\section{Nematostella and sea urchin whole mount in situ hybridization with locked nucleic acids probes}

Fixations and hybridizations of Nematostella carried out as described in ref. ${ }^{28}$ and for sea urchin larvae done as described in ref. ${ }^{29}$ with the following adaptations: content of formamide in hybridization mix increased to $70 \%$ instead of $50 \%$. Pre-hybridization and hybridization carried out at $37^{\circ} \mathrm{C}$ instead of $60{ }^{\circ} \mathrm{C}$.

\section{Microscopy}

Nitroblue tetrazolium chloride (NBT)/5-Bromo-4-chloro-3-indolyl-phosphate (BCIP) stained embryos were imaged using a Leica TCS SPE confocal microscope through reflection imaging as described in ref. ${ }^{27}$. White light pictures were taken under Nomarski optics using a Zeiss Axiophot microscope. 


\section{microRNA cloning and sequencing}

Small RNA cloning was performed as described in ref. ${ }^{30}$. Small RNA libraries were sequenced using the Illumina platform. We only analysed sequence reads with quality score higher than 35 in Solexa/Illumina files. Reads were clustered according to sequence similarity and of these, dominant reads were analysed by BLAST search against miRBase v10.0. Comparison required $\geq 80 \%$ identity of the query with the target and $\geq 7$ out of 8 nucleotides in the $5^{\prime}$ end and yielded a list of conserved microRNAs (Supplementary Table 1).

Full Methods and any associated references are available in the online version of the paper at www.nature.com/nature.

\section{Supplementary Material}

Refer to Web version on PubMed Central for supplementary material.

\section{Acknowledgments}

We thank A. Fischer for drawing schematic illustrations and providing probes for tropomyosin 1, A. Boutla for advice when initiating the project, J. Brennecke for help with small RNA cloning and discussions, M. Hentze for critical reading of the manuscript, P. Steinmetz and U. Technau for Nematostella embryos and discussions, E. Arboleda and I. Arnone for sea urchin plutei and discussions. V. Benes and the EMBL-Genecore facility for expert technical advice, W. R. McCombie, M. Rooks and E. Hodges for help with sequencing and M. Arumugam, V. Van Noort, J. Muller and C. Creevey for advice in target analysis.

\section{References}

1. Grimson A, et al. Early origins and evolution of microRNAs and Piwi-interacting RNAs in animals. Nature 2008;455:1193-1197. [PubMed: 18830242]

2. Wheeler BM, et al. The deep evolution of metazoan microRNAs. Evol. Dev 2009;11:50-68. [PubMed: 19196333]

3. Raible F, et al. Vertebrate-type intron-rich genes in the marine annelid Platynereis dumerilii. Science 2005;310:1325-1326. [PubMed: 16311335]

4. Tessmar-Raible K, et al. Conserved sensory-neurosecretory cell types in annelid and fish forebrain: insights into hypothalamus evolution. Cell 2007;129:1389-1400. [PubMed: 17604726]

5. Shkumatava A, Stark A, Sive H, Bartel DP. Coherent but overlapping expression of microRNAs and their targets during vertebrate development. Genes Dev 2009;23:466-481. [PubMed: 19240133]

6. Reinhart BJ, et al. The 21-nucleotide let-7 RNA regulates developmental timing in Caenorhabditis elegans. Nature 2000;403:901-906. [PubMed: 10706289]

7. Caygill EE, Johnston LA. Temporal regulation of metamorphic processes in Drosophila by the let-7 and $m i R-125$ heterochronic microRNAs. Curr. Biol 2008;18:943-950. [PubMed: 18571409]

8. Sokol NS, Xu P, Jan YN, Ambros V. Drosophila let-7 microRNA is required for remodeling of the neuromusculature during metamorphosis. Genes Dev 2008;22:1591-1596. [PubMed: 18559475]

9. Poy MN, et al. A pancreatic islet-specific microRNA regulates insulin secretion. Nature 2004;432:226230. [PubMed: 15538371]

10. Wienholds E, et al. MicroRNA expression in zebrafish embryonic development. Science 2005;309:310-311. [PubMed: 15919954]

11. Scholz CB, Technau U. The ancestral role of Brachyury: expression of NemBral in the basal cnidarian Nematostella vectensis (Anthozoa). Dev. Genes Evol 2003;212:563-570. [PubMed: 12536320]

12. Arendt D, Technau U, Wittbrodt J. Evolution of the bilaterian larval foregut. Nature 2001;409:8185. [PubMed: 11343117]

13. Nielsen, C. Animal Evolution: Interrelationships of the Living Phyla. 2nd edn. Oxford Univ. press; 2001.

14. Kapsimali M, et al. MicroRNAs show a wide diversity of expression profiles in the developing and mature central nervous system. Genome Biol 2007;8:R173. [PubMed: 17711588] 
15. Deo M, et al. Detection of mammalian microRNA expression by in situ hybridization with RNA oligonucleotides. Dev. Dyn 2006;235:2538-2548. [PubMed: 16736490]

16. Farh KK, et al. The widespread impact of mammalian microRNAs on mRNA repression and evolution. Science 2005;310:1817-1821. [PubMed: 16308420]

17. Aboobaker AA, et al. Drosophila microRNAs exhibit diverse spatial expression patterns during embryonic development. Proc. Natl Acad. Sci. USA 2005;102:18017-18022. [PubMed: 16330759]

18. González-Estévez C, et al. Diverse miRNA spatial expression patterns suggest important roles in homeostasis and regeneration in planarians. Int. J. Dev. Biol. 2009 (in the press).

19. Denes AS, et al. Molecular architecture of annelid nerve cord supports common origin of nervous system centralization in bilateria. Cell 2007;129:277-288. [PubMed: 17448990]

20. Rao PK, et al. Myogenic factors that regulate expression of muscle-specific microRNAs. Proc. Natl Acad. Sci. USA 2006;103:8721-8726. [PubMed: 16731620]

21. Sood P, et al. Cell-type-specific signatures of microRNAs on target mRNA expression. Proc. Natl Acad. Sci. USA 2006;103:2746-2751. [PubMed: 16477010]

22. Prochnik SE, Rokhsar DS, Aboobaker AA. Evidence for a microRNA expansion in the bilaterian ancestor. Dev. Genes Evol 2007;217:73-77. [PubMed: 17103184]

23. Szafranska AE, et al. MicroRNA expression alterations are linked to tumorigenesis and non-neoplastic processes in pancreatic ductal adenocarcinoma. Oncogene 2007;26:4442-4452. [PubMed: 17237814]

24. Pasquinelli AE, et al. Conservation of the sequence and temporal expression of let-7 heterochronic regulatory RNA. Nature 2000;408:86-89. [PubMed: 11081512]

25 . Vigh B, et al. The system of cerebrospinal fluid-contacting neurons. Its supposed role in the nonsynaptic signal transmission of the brain. Histol. Histopathol 2004;19:607-628. [PubMed: 15024719]

26. Harada Y, et al. Developmental expression of the hemichordate otx ortholog. Mech. Dev 2000;91:337-339. [PubMed: 10704860]

27. Jékely G, Arendt D. Cellular resolution expression profiling using confocal detection of NBT/BCIP precipitate by reflection microscopy. Biotechniques 2007;42:751-755. [PubMed: 17612299]

28. Rentzsch F, et al. Asymmetric expression of the BMP antagonists chordin and gremlin in the sea anemone Nematostella vectensis: implications for the evolution of axial patterning. Dev. Biol 2006;296:375-387. [PubMed: 16828077]

29. Arenas-Mena C, Cameron AR, Davidson EH. Spatial expression of Hox cluster genes in the ontogeny of a sea urchin. Development 2000;127:4631-4643. [PubMed: 11023866]

30. Pfeffer S, et al. Identification of microRNAs of the herpesvirus family. Nature Methods 2005;2:269276. [PubMed: 15782219] 


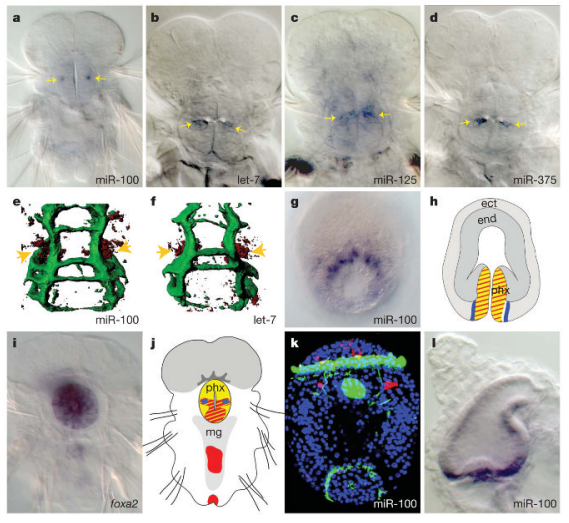

Figure 1. Foregut-related expression of conserved microRNAs

a, Ventral view of 72 hours post fertilization (h.p.f.) Platynereis. b-d, dorsal view of 5 d.p.f. Platynereis. e, f, Three-dimensional reconstructions of 5 d.p.f. foregut (acTub green, miR-100 and let-7 red). g, Oral view of Nematostella planula. $\mathbf{h}$, Scheme of Nematostella planula with pharynx showing brachyury ${ }^{11}$ (red) foxa ${ }^{11}$ (yellow) and miR-100 (blue) expression (phx, pharynx; end, endoderm; ect, ectoderm). i, Ventral view of 72 h.p.f. Platynereis. j, scheme of 72 h.p.f. Platynereis foregut expressing foxa (yellow), brachyury ${ }^{12}$ (red) and miR-100 (blue). $\mathbf{k}$, Ventral view of Capitella stage 4 larva with expression lateral to stomodaeum and in brain. l, Lateral view of Strongylocentrotus with expression in sphincter between oesophagus/ stomach. 


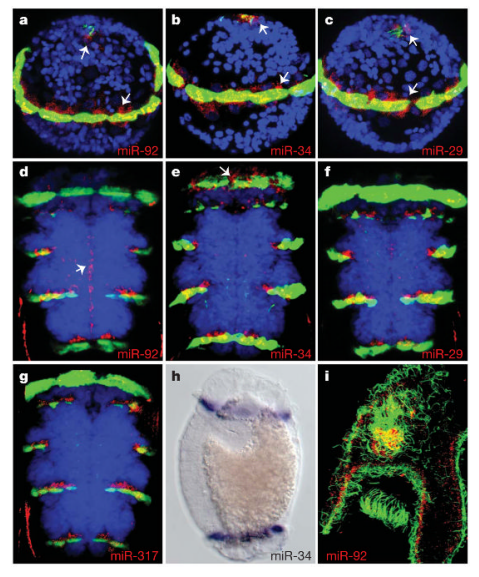

Figure 2. MicroRNAs expressed in locomotor ciliated cells a-c, Ventral-anterior views of 24 h.p.f. Platynereis trochophora larvae with microRNA expression (red) in prototroch cells and apical organ (arrowheads) (blue, DAPI; green, acTub). d, Ventral view of 72 h.p.f. Platynereis with expression in ciliated cells and midline (arrow). e, 72 h.p.f. Platynereis; expression in ciliated cells of trunk and prototroch (arrow). f, $\mathbf{g}, 72$ h.p.f. Platynereis; expression in ciliated cells of the trunk. h, Lateral view of Capitella stage 6 larva; expression in ciliated bands. i, Ventral-lateral view of Strongylocentrotus pluteus larva with expression in ciliated cells lining the foregut and arms. 


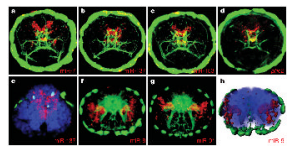

Figure 3. Expression of brain-specific microRNAs

a-d, Apical view of 48 h.p.f. Platynereis with expression in dorso-medial brain (green, acTub; red, microRNAs). e, Apical view of 72 h.p.f. Platynereis brain section showing colocalization of microRNA (red) with serotonin (cyan) (blue, DAPI, green, acTub). f, g, Apical views of 5 d.p.f. Platynereis brain. h, 3D reconstruction of 5 d.p.f. apical brain with superficial expression at the base of antennae. 

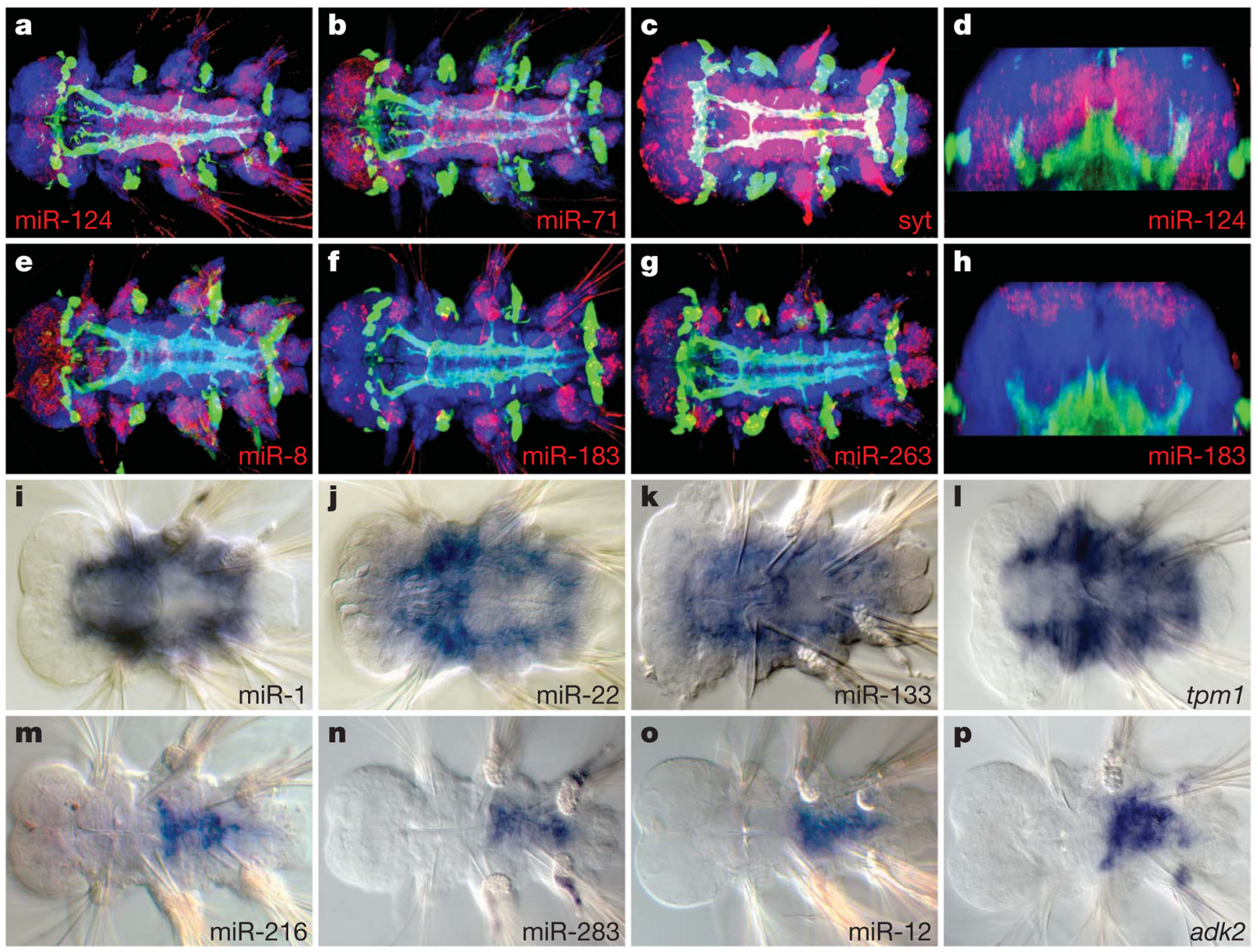

Figure 4. MicroRNAs demarcating organ systems

a, b, Ventral views of 5 d.p.f. Platynereis with expression in CNS (blue, DAPI; green, acTub; red, microRNA). c, synaptotagmin (syt) expression at 5 d.p.f. ventral view. d, Virtual section of 5 d.p.f. Platynereis brain exemplifying the basal expression of CNS-specific microRNAs. $\mathbf{e}-\mathbf{g}$, Ventral views of 5 d.p.f. worms with expression in peripheral nervous system. $\mathbf{h}$, Section of 5 d.p.f. Platynereis brain exemplifying the apical expression of peripheral nervous systemspecific microRNAs. i-l, Ventral views of 72 h.p.f. Platynereis with expression in differentiated musculature. tpm, Tropomyosin. $\mathbf{m}-\mathbf{p}$, dorsal views of 5 d.p.f. Platynereis with expression in differentiating midgut. adk, Adenosine kinase. 